ZIBELINE INTER S

\title{
A DESIGN OF HEART RATE MONITOR BRACELET BASED ON BP NEURAL NETWORK
}

\author{
Yilun Liao' ${ }^{1}$, Yu Zhang², Xiaohui Cheng2*, Yanping Kang² \\ ${ }^{1}$ Guilin Zhishen Information Technology Limited company. \\ ${ }^{2}$ Institute of Information Science and Engineering, Guilin University of Technology. \\ *Corresponding Authors Email: cxiaohui@glut.edu.cn
}

This is an open access article distributed under the Creative Commons Attribution License, which permits unrestricted use, distribution, and reproduction in any medium, provided the original work is properly cited

\section{ARTICLE DETAILS}

Article History:

Received 12 November 2017

Accepted 12 December 2017

Available online 1 January 2018

\section{ABSTRACT}

Article History: Through BP neural network self-learning, the wearable heart rate test bracelet is self-learned to determine the state of the heart in the current time period, and to pre-warn the abnormality of the heart. And when an abnormal heart rate is found, an alarm is immediately issued to reduce the risk of heart problems to people.

\section{KEYWORDS}

BP neural network, hand ring model, heart rate monitoring.

\section{INTRODUCTION}

In today's society, people are increasingly paying attention to the health of the body. However, when people do physical exercises, they may sometimes cause unexpected accidents such as sudden death because they do not know their own conditions. Nowadays, most of the heart rate monitoring wristbands on the market can only perform real-time heart rate detection and data recording and cannot perform corresponding data analysis and autonomous analysis to predict the probability of heart problems [1]. The ability of autonomous learning in the BP neural network can be based on previous heart rate monitoring data and feedback learning to compare, draw results, predict possible dangerous conditions, and send messages to the user and monitor of the bracelet in time. The risk is minimized.

\section{BP NEURAL NETWORK MODEL}

BP neural network is a multi-layer feedforward neural network consisting of input layer, hidden layer and output layer. Figure 1 shows the structure of a typical three-layer BP neural network. Each layer and each layer are interconnected. There is no connection between the same layers. The number of hidden layers is not determined.

There are two types of signal flow between layers, one is a working signal, and the other is an output signal that is actually generated when the input signal is propagated from the input layer to the output end and is a function of the input and the weight [2]. The other is the error signal, which is the difference between the actual output and the expected output of the network. Therefore, the basic BP neural network algorithm has two directions, namely the forward propagation of the signal and the backward propagation of the error. When the actual output is calculated, the forward propagation direction of the signal is calculated; when the weight and the threshold correction are performed, the direction of the backward propagation is calculated [3].

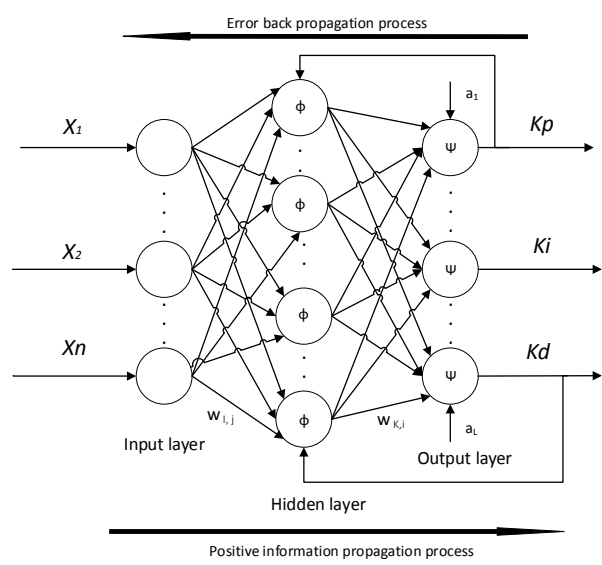

Figure 1: Typical three-layer BP neural network structure diagram

In Figure $1, x_{i}=\left(x_{1}, x_{2}, \cdots, x_{n}\right)$ is the input of the number I's node of the input layer of the BP neural network; $i=1,2,3 \ldots n$; $w_{i, j}$ is the input layer I The connection weights between the node and the number j's node of the hidden layer; $w_{k, i}$ is the connection weight between the i node of the hidden layer and the kth node of the output layer; $a_{k}$ is the number k's node of the output layer. Threshold; $\Phi$ represents the incentive function of the hidden layer; $\Psi$ represents the excitation function of the output layer; $\mathrm{k}_{\mathrm{d}}$ represents the output of the number k's node of the output layer [4]. In forward propagation, the input signal vector $\mathrm{x}_{\mathrm{i}}$ is transmitted layer by layer from the input layer through the hidden layer and finally to the 
output layer, and is calculated by connecting the weight vector, the threshold vector, and the corresponding excitation function of each layer. The predicted output value $y$ of the output layer is obtained. If there is an error between the predicted value $\mathrm{k}$ and the target value $\mathrm{K}$, the error portion is reversed and transmitted layer by layer, and the weights and thresholds of the connections of the network layers are adjusted along the error reduction direction. Repeat the above process and adjust the weights and thresholds of each layer of the network one by one so that the predicted values of the BP neural network continue to approach the actual output values until the entire learning process is over.

The relationship between input and output of BP algorithm is:

Input $=$ net $=\mathrm{x}_{1} \mathrm{w}_{1}+\mathrm{x}_{2} \mathrm{w}_{2}+\cdots+\mathrm{x}_{\mathrm{n}} \mathrm{w}_{\mathrm{n}}$

Output=y $=\mathrm{f}($ net $)=\frac{1}{1+e^{-n e t}}$

The derivative of the output

$\mathrm{f}^{\prime}($ net $)=\frac{1}{1+e^{-n e t}}-\frac{1}{\left(1+e^{-n e t}\right)^{2}}=\mathrm{y}(1-\mathrm{y})$

\section{FORECAST MONITORING ALGORITHM}

\subsection{Hand ring model}

The wristband is a portable wearable device. As shown in Figure2, a display panel, a built-in chip, and a corresponding circuit form a wristband main body. The wristband is a plastic or rubber product. A heart rate test module is arranged on the back of the display to complete the entire heart rate. Testing process.

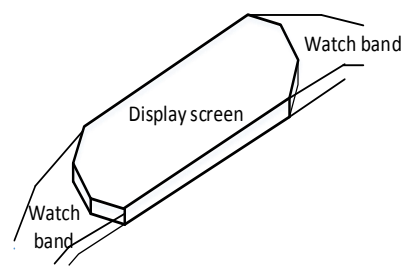

Figure 2: bracelet hardware model

\subsection{Algorithm flow}

The heart rate prediction monitoring system uses the built-in chip of the wristband to store and remember the heart rate data, and then analyzes and analyzes the heart rate data. The system has a built-in timing function. When the heart rate is within the normal range, the user's different heart rates are recorded at different time periods. The BP algorithm is used to autonomously learn and analyze the user's heart rate fluctuation range and to perform memory. The system also has a mode adjustment function [5]. When the user performs a movement, it adjusts to a movement mode. The chip records the corresponding heart rate data, and autonomously learns and calculates the heart rate data of the user's exercise mode through the BP algorithm, and performs learning and memory. At the same time, daily life mode, work mode, sleep mode and other modes that users can adjust themselves are also set. After a period of learning and memory, when the wristband detects that the user's heart rate is different from the user's mode, or the heart rate suddenly fluctuates, the alarm system will automatically send an alarm to the user of the wristband and the tester to ensure that the user gets Effective monitoring and timely treatment. As shown in Figure 3 is the bracelet alarm process.

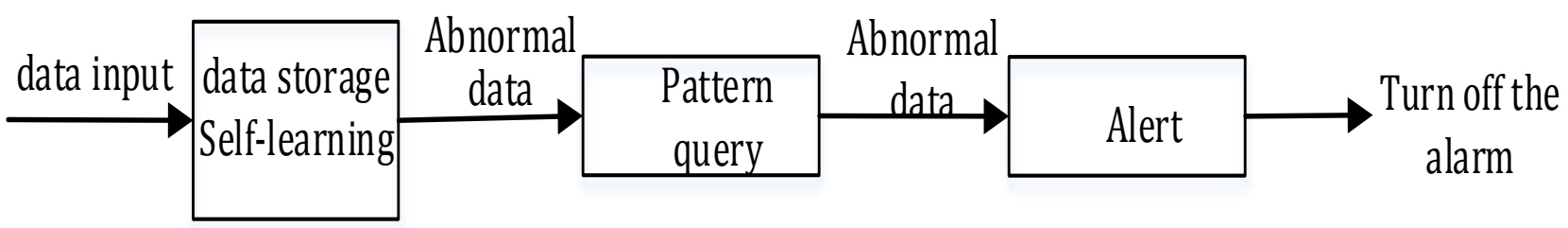

Figure 3: Hand ring alarm flow chart

\subsection{BP algorithm steps}

(1) Bracelet initialization

According to the performance of the wristband itself, a random number within the interval $(-1,1)$ is assigned to each connection weight value, and an error function e is set to provide a calculation accuracy value $\varepsilon=0.02$ 0.05 and a maximum number of learning times $\mathrm{N}=50$.

(2) Randomly select the kth heart rate input sample and corresponding expected output.

$y o_{0}(k)=f\left(y i_{0}(k)\right) \quad o=1,2, L p$

(3) Heart rate test signal forward propagation process Calculate heart rate signal input and output

$h i_{h}(k)=\sum_{i=1}^{n} w_{i h} x_{i}(k)-b_{h} h=1,2, L p$

$h o_{h}(k)=f\left(h i_{h}(k)\right) \quad h=1,2, L p$

$y i_{0}(k)=\sum_{h=1}^{p} w_{h 0} h o_{h}(k)-b_{0} o=1,2, L p$

$y o_{0}(k)=f\left(y i_{0}(k)\right) \quad o=1,2, L p$

(4) Test error back propagation process

Using the desired heart rate signal output and the actual heart rate output, the partial derivative $\delta_{0}(\mathrm{k})$ of the error function to each neuron of the output layer is calculated.

$\frac{\partial \mathrm{e}}{\partial \mathrm{y} i_{o}}=\frac{\partial\left(\frac{1}{2} \sum_{o=1}^{q}\left(d_{o}(k)-y o_{o}(\mathrm{k})\right)\right)^{2}}{\partial y i_{o}}$

$=-\left(d_{o}(k)-y o_{o}(k)\right) y o^{\prime}{ }_{o}(k) 3-5$ $=-\left(d_{o}(k)-y o_{o}(k)\right) f^{\prime}\left(y i_{o}(k)\right) @-\delta_{o}(k)$

(5) The partial derivative of the error function for each neuron of the hidden layer, $\delta_{\mathrm{h}}(\mathrm{k})$, is calculated using the connection weight from the hidden layer to the output layer, the output $\delta_{0}(\mathrm{k})$, and the output of the hidden layer.

$\frac{\partial \mathrm{e}}{\partial w_{h o}}=\frac{\partial e}{\partial y i_{o}} \frac{\partial y i_{o}}{\partial w_{h o}}=-\delta_{o}(k) h o_{h}(k)$

$\frac{\partial \mathrm{e}}{\partial w_{i h}}=\frac{\partial e}{\partial h i_{h}(k)} \frac{\partial h i_{h}(k)}{\partial w_{i h}}$

$\frac{\partial \mathrm{h} i_{h}(k)}{\partial w_{i h}}=\frac{\partial\left(\sum_{i=1}^{n} w_{i h} x_{i}(k)-b_{h}\right)}{\partial w_{i h}}=x_{i}(k)$

$\frac{\partial \mathrm{e}}{\partial h i_{h}(\mathrm{k})}=\frac{\partial\left(\frac{1}{2} \sum_{o=1}^{q}\left(d_{o}(k)-y o_{o}(k)\right)^{2}\right)}{\partial h o_{h}(k)} \frac{\partial h o_{h}(k)}{\partial h i_{h}(k)}$

$=\frac{\partial\left(\frac{1}{2} \sum_{o=1}^{q}\left(d_{o}(k)-f\left(y i_{o}(k)\right)\right)^{2}\right)}{\partial h o_{h}(k)} \frac{\partial h o_{h}(k)}{\partial h i_{h}(k)}$

$=\frac{\partial\left(\frac{1}{2} \sum_{o=1}^{q}\left(d_{o}(k)-f\left(\sum_{h=1}^{p} w_{h o} h o_{h}(k)-b_{o}\right)^{2}\right)\right)}{\partial h o_{h}(k)} \frac{\partial h o_{h}(k)}{\partial h i_{h}(k)}$

$=-\sum_{o=1}^{q}\left(d_{o}(k)-y o_{o}(k)\right) f^{\prime}\left(y i_{o}(k)\right) w_{h o} \frac{\partial h o_{h}(k)}{\partial h i_{h}(k)}$

$=-\left(\sum_{o=1}^{q} \delta_{o}(k) w_{h o}\right) f^{\prime}\left(h i_{h}(k)\right) @-\delta_{h}(k)$

(6) The connection weight $\mathrm{W}_{\mathrm{ho}}(\mathrm{k})$ is corrected by using the output of each neuron of $\delta_{0}(\mathrm{k})$ and the hidden layer neurons.

$\Delta w_{h o}(k)=-\mu \frac{\partial e}{\partial w_{h o}}=\mu \delta_{o}(k) h o_{h}(k)$

$w_{h o}^{N+1}=w_{h o}^{N}+\eta \delta_{o}(k) h o_{h}(k)$ 
(7) Correct the connection weights using the input of each neuron in the hidden layer $\delta_{\mathrm{h}}(\mathrm{k})$ and input neurons.

$$
\begin{aligned}
\Delta w_{i h}(k) & =-\mu \frac{\partial e}{\partial w_{h i}} \\
& =-\mu \frac{\partial e}{\partial h i_{h}(k)} \frac{\partial h i_{h}(k)}{\partial w_{i h}}=\delta_{h}(k) x_{i}(k)
\end{aligned}
$$

$w_{i h}^{N+1}=w_{i h}^{N}+\eta \delta_{h}(k) x_{i}(k)$

(8) Calculate the global error E

$\mathrm{E}=\frac{1}{2 m} \sum_{k=1}^{m} \sum_{o=1}^{q}\left(d_{o}(k)-y_{o}(k)\right)^{2}$

(9) Determine whether the error meets the bracelet alarm accuracy requirement. The algorithm ends when the error reaches the preset accuracy of 0.03-0.05 or the number of learning is greater than 50 . Otherwise, the next data sample and the corresponding expected output are selected, and the learning is continued until the target result is obtained.

\section{SUMMARY}

Based on the BP neural network algorithm, this article designs a wristband that can monitor and warn the abnormal heart rate. It can collect, store and analyze the user's heart rate, conduct self-learning, predict the possible problems of the user's heart rate, and perform timely alarm. The advantage of using a neural network is that there is no need to design any mathematical model. It is only necessary to learn, and process data based on past experience for data prediction, classification, pattern recognition and other data processing applications. In most cases, the results obtained using the neural network algorithm are superior to the traditional mathematical statistics methods and can be applied to occasions where there are many influencing factors and large variations. Through the above research, it is possible to use the neural network BP algorithm to alert the ring user's heart rate problem, and the neural network can be applied to this.

\section{ACKNOWLEDGMENTS}

As the research of the thesis is sponsored by Key R \& D projects of Guangxi Science and Technology Program (AB17195042), Major scientific research project of Guangxi higher education (No:201201ZD012), Guangxi Key Laboratory Fund of Embedded Technology and Intelligent System., we would like to extend our sincere gratitude to them.

\section{REFERENCE}

[1] Wanzhi, C., Dongzhe, L. 2018. Industrial Control Network Intrusion Detection Method Combining Whitelist Filtering and Neural Networks [J]. Computer Applications, 2, 363-369.

[2] Xia, S., Long, W., Daojun, H. 2016. Application of artificial bee colony optimization BP neural network in intrusion detection [J]. Computer Engineering, 42 (2), 190-194.

[3] Junsong, L. 2017. Research on BP algorithm based on neural network and its application in network intrusion detection [J]. Modern Electronic Technology, 2 (7), 20-35.

[4] Bin, C., Li, H. 2016. Simulation Research Based on BP Neural Network Algorithm [J]. Information Technology, 8, 85-92.

[5] Markopoulos, A.P., Georgiopoulos, S., Manolakos, D. 2016. On the use of back propagation and radial basis function neural networks in surface roughness prediction [J]. Journal of Industrial Engineering International, 3, 65-73. 